\title{
HETEROCYCLIC SYNTHESIS WITH ACTIVATED NITRILES: AND EXPEDITUS SYNTHETIC APPROACH TO POLYFUNCTIONALLY SUBSTITUTED HETEROCYCLO PYRIMIDINES, PYRAZOLO, CHROMENO AND TETRAHYDROBENZO[B]THIOPHENE
}

\author{
Mohamed. A. Elian, Ibrahim. S. A. Hafiz, Mohamed. A. M. Abdel Reheim \\ Department of Chemistry, Faculty of Education, Suez Canal University, Arish, Egypt.
}

\begin{abstract}
New derivatives of thioxo-2, 5-dihydro-1H-pyrano [2,3-d] pyrimidine 3 was obtained from the reaction of compound 1a with acetic anhydride. Compound 1a was treated with acetic anhydride for 5 min and (or) acid chloride to yield the corresponding pyrano [2,3-d] pyrimidin-7-yl-acetamide 5a and pyrano [2,3-d] pyrimidin-7-yl benzamide $5 \mathrm{~b}$ derivatives. Treatment of compound 1a with bifunction compounds such as hydrazine hydrate, hydroxylaminehydrochloride, orthophenylene diamine, urea and ammonium thiocyanate to give compounds $6,8,10$ and 12 respectively. Fusion of isolated compounds $6,8,10$ and 12 in basic medium afforded the condensed pyrazolo, chromeno, pyrimido and thiopyrimidine 7, 9, 11 and 13 respectively. Treatment of compound $1 \mathrm{~b}$ with ethylcyanoacetate afforded cyanoacetamide derivatives 15 . The reactivity of compound 15 towards some electrophilic and nucleophilic reagents was also investigated. The structures of the products and conceivable mechanisms are discussed; the newly synthesized compounds were characterized by IR, 1H-NMR and mass spectral studies.
\end{abstract}

Key Words: Pyranopyridine, pyranopyrimidine and thioxopyrimidine

\section{INTRODUCTION}

Several derivatives of the pyrane or of fused pyrane ring systems are endowed with different types of biological activities. It has been reported that pyrane derivatives have attracted a great deal of interest owing to their antimicrobial activity (El-Agrody, et al, 2001; Bedair, et al, 2000; El-Agrody, et al, 2000), inhibition of influenza, virus sialidases (Taylor, et al, 1998), mutagenic activity (Hirmoto, et al, 1997), antiviral (Martnez and marco, 1997) and antiproliferaction agents (Dell and smith, 1993), sex-pheromones (Bianchi and Tava, 1987), antitumor (Eiden and Denk, 1991) anti-inflammatory agents (Shishoo et al., 1981). Moreover pyrane derivatives are well known for antifungual and plant growth stimulating effects (Ohira and yatagai, 1993), central nervous system (CNS) activity (Elden and Denk, 1991) and hypotensive effect (Tandom, et al, 1991).
In view of these observations and in continuation of our previous work in heterocyclic chemistry, we report here on the use of 7-amino-5-(4-chlorophenyl)-4-phenyl-2thioxo-2, 5-dihydro-1H-pyrano [2,3-d] pyrimidine-6-carbonitrile 1a and 7-amino-4,5diphenyl-2-thioxo-2,5-dihydro-1H-pyrano [2,3d] pyrimidine-6-carbonitrile $\mathbf{1 b}$ as a key intermediate for the synthesis of new series of pyranopyrimidine, pyrano [2,3-d]pyrimidine benzamide, chromeno [2,3-e] diazpin derivatives, pyrano [2,3-d] pyrimidine-7-yl-3(dimethylamino) acrylamide and pyrano [2,3d]pyrimidin-7-yl-1H-pyrazole-4-carboxamide derivatives.

\section{RESULTS AND DISCUSSIONS}

This work is aimed to the synthesis of new compounds related to $\beta$ enaminonitriles of pyrano [2,3-d] pyramidine derivatives. We have found 
that 1a is an attractive starting material for the preparation of some new fused heterocyclic compounds. Thus, it has been found that 1a reacted with acetic anhydride for a long time to give thioxo2,5-dihydro-1H-pyrano[2,3-d]pyrimidine $\mathbf{3}$ in a quantitative yield. The structure of $\mathbf{3}$ was based on its spectral analysis. The expected pyrimidine $\mathbf{2}$ formation was ruled out on the basis of analytical and spectral data (El-Gaby, et al, 2006) (Scheme 1).

Also, the acetamide derivatives $\mathbf{5 a}$ was achieved by the refluxing of compound 1a with acetic anhydride for $5 \mathrm{~min}$. the structure of compound 5a was confirmed from its elemental and spectral analysis. Also, $\quad$ pyrano[2,3-d]pyrimidin-7-ylacetamide 5a and pyrano[2,3-d]pyrimidin7-yl-benzamide $\mathbf{5 b}$ were obtained by reaction of compound $\mathbf{1 a}$ with acid chloride derivatives in pyridine at reflux temperature [Siham, 2012; El-Sharkawy, et al, 2012; Fathy, et al, 2004]. Compound 1a was allowed to react with hydrazine hydrate in ethanol to give 6. Similar to this reaction, reaction of compound 1a with bifunction amino compounds such as orthophenylene diamine, urea and ammonium thiocyanate. Assignment of structure as 8, 10 and 12 were confirmed on the basis of their correct elemental analysis as well as compatible spectral data. While fusion of isolated intermediate 6, 8, 10 and 12 in ethanolic sodium ethoxide solution furnished the condensed pyrazolo, chromeno, pyrimido and thiopyrimidine $\mathbf{7}, \mathbf{9}, \mathbf{1 1}$, and $\mathbf{1 3}$ respectively. The ${ }^{1} \mathrm{H}-\mathrm{NMR}$ spectrum of compounds 7, 9, 11 and 13 are in agreement with proposed structures. Also, Compound 7 was obtained by reaction of 1 a with hydroxylamine hydrochloride (EIGaby, et al, 2006; Hatem, et al, 2005; Mohammed, et al; 1989; Sherif and Hussein, 1997; Ahmed, et al; Gamal, et al, 2005; Mohammed, et al; 2009, Said, et al, 2004) (scheme 2).

In continuation of our interest in developing the synthesis of polyfunctionally substituted heteroaromatics, we report here on the utility of $\mathbf{1 b}$ as aprecursor for the synthesis of polyfunctionally substituted pyranopyrimidine, $\mathrm{N}$-phenylacetohydrazonyl cyanide and cyclohexlideneacetamide derivatives. Some of the obtained products seem promising for further chemical transformations. Thus, a trial to prepare the 6-amino-7-cyano-4, 5diphenyl-2-thioxo-5, 9-dihydro-2H-pyrimido $\left[5^{\prime}, 4^{\prime}: 5,6\right]$ pyrano $[2,3-\mathrm{d}]$ pyrimidine- $8-(1 \mathrm{H})-$ one $\mathbf{1 4}$ through the interaction between $\mathbf{1 b}$ with ethylcyanoacetate in basic medium was unfortunately unsuccessful, but led interesting to compound $\mathbf{1 5}$ which formed as the reactants were mixed together in amount of sodium ethoxide. The actual structure of $\mathbf{1 5}$ was confirmed for the reaction product on the basis of their elemental and spectral data (Salman, 1999; Samir, et al, 2009; Haider, et al, 2012) (scheme 3).

The cyanoacetamide derivatives $\mathbf{1 5}$ react with dimethylformamidedimethylacetal (DMF-DMA) to yield the corresponding enaminone 16 in high yield. The structure of the latter product was established on the basis of its elemental analysis and spectral data (Samir, et al, 2009; Haider, et al, 2012; Mohamed and Khaled, 2009). Compound 16 underwent an interamolecular heterocyclization upon boiling in hydrazine hydrate to afford aminopyrazole derivatives 17. The structure of compound $\mathbf{1 7}$ was confirmed for the reaction product on the basis of their elemental and spectral data Mohamed and [Haider, et al, 2012; Khaled, 2009]. In a similar manner, pyrano [2,3-d]pyrimidin-7-yl)-1Hpyrazole- 4- carboxamide derivatives 22 could be prepared by the reaction of enaminone 16 with malononitrile in refluxing ethanol.

The structure of $\mathbf{2 2}$ was based on its spectral analysis. Compound 22 was formed via Dimruth rearrangement (Haider, et al, 2012) illustrated in (scheme 4).

Furthermore, the behaviour of cyanoacetamide 15 towards some electrophilic reagent such as arylidenemalononitrile was also investigated. Thus, compound 15 was reacted with 4chlorobenzylidenemalononitrile $\mathbf{2 3}$ in refluxing ethanol/Pip to give 
SINAI Journal of Applied Sciences (ISSN: 2314-6079), Vol.:(3), Is.:(2), Aug. 2014
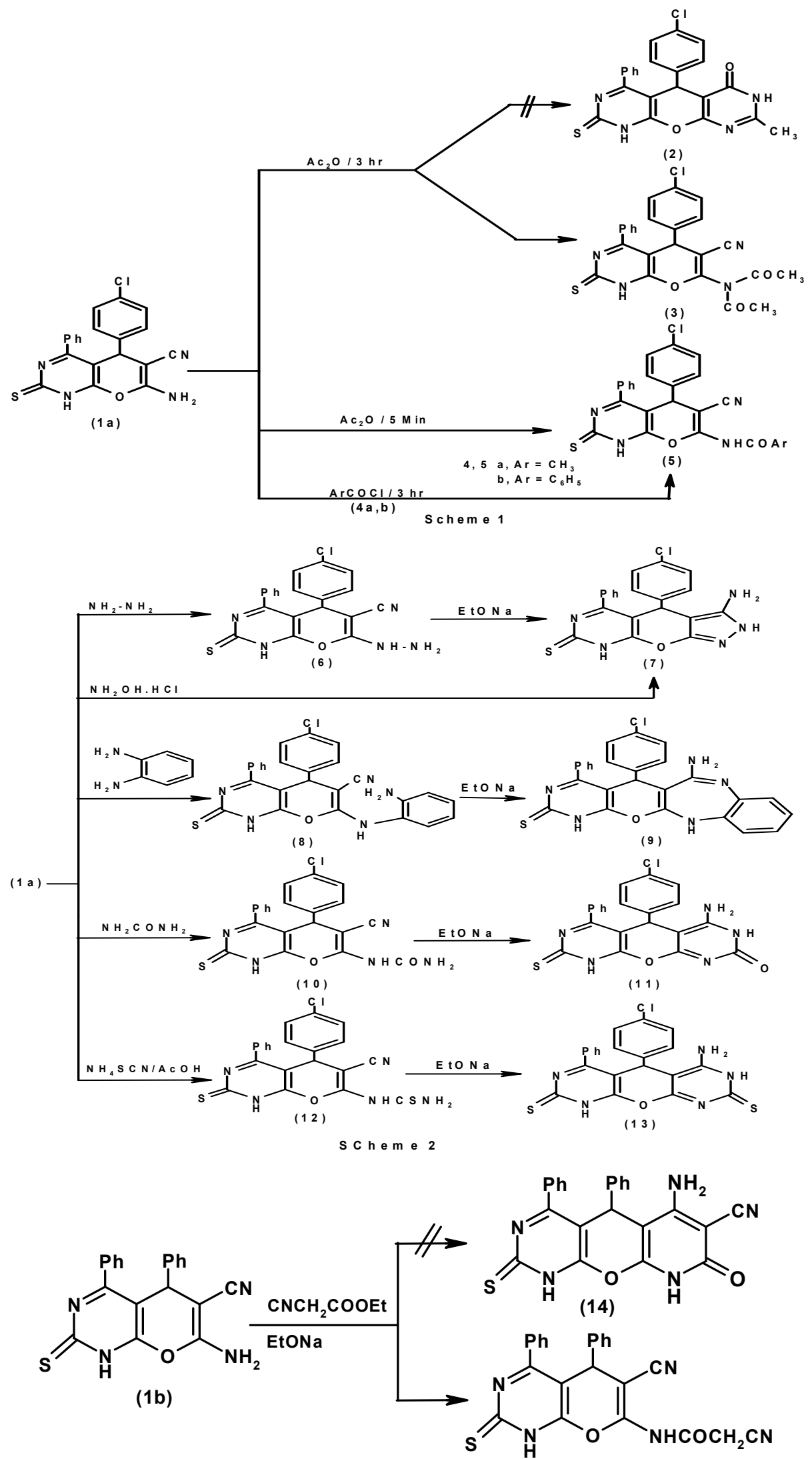

Scheme 3

(15) 
pyrmidinecarbonitrile derivatives $\mathbf{2 8}$. The coupling of 15 with arylidenediazonium chlorides gave the aryl hydrazones 30a-c (Haider, et al, 2012). On the other hand, the reaction of $\mathbf{1 5}$ with cyclohexanone in ethanol/Pip afforded a product $\mathbf{3 1}$. Compound 31 was confirmed by spectroscopic data and its chemical reactivity of this molecule to Geweld reaction with elemental sulfur. So, further reaction of compound $\mathbf{3 1}$ with elemental sulfur in refluxing DMF in the presence of little amount of piperidine afforded 32 . Compound $\mathbf{3 2}$ was established by analytical spectra data and elemental analysis (scheme 5).

\section{EXPERIMENTS}

All melting points were measured using Akofler Block instrument and are uncorrected. IR spectra $(\mathrm{KBr})$ were recorded on a FTIR 5300 spectrometer (v, $\left.\mathrm{cm}^{-1}\right)$. The ${ }^{1} \mathrm{H}-\mathrm{NMR}$ spectra were recorded in $\mathrm{DMSO}_{-} \mathrm{d}_{6}, \mathrm{CDCl}_{3}$ at $300 \mathrm{MHz}$ on a Varian Gemini NMR. 1000 EX mass spectrometer at $70 \mathrm{ev}$. The purity of synthesized compounds was checked by thin layer chromatography TLC (aluminum sheets) using n-hexane, ethyl acetate $(9: 1, \mathrm{~V} / \mathrm{V}, \quad 7: 3 \mathrm{~V} / \mathrm{V})$ eluent. Elemental analyses were carried out by<smiles>N#CC1=C(N=CC=O)Oc2[nH]c(=S)nc(-c3ccccc3)c2C1c1ccccc1</smiles>

(15)<smiles>N#CC1=C(NC=O)Oc2[nH]c(=S)nc(-c3ccccc3)c2C1c1ccccc1</smiles>

(16) $\mathrm{NH}_{2} \mathrm{NH}_{2}$<smiles>CN(C)CC(N)=NC1=C(C#N)C(c2ccccc2)c2c(-c3cn[nH]c3N)nc(=S)[nH]c2O1</smiles>

(17)

Scheme 4

(15)

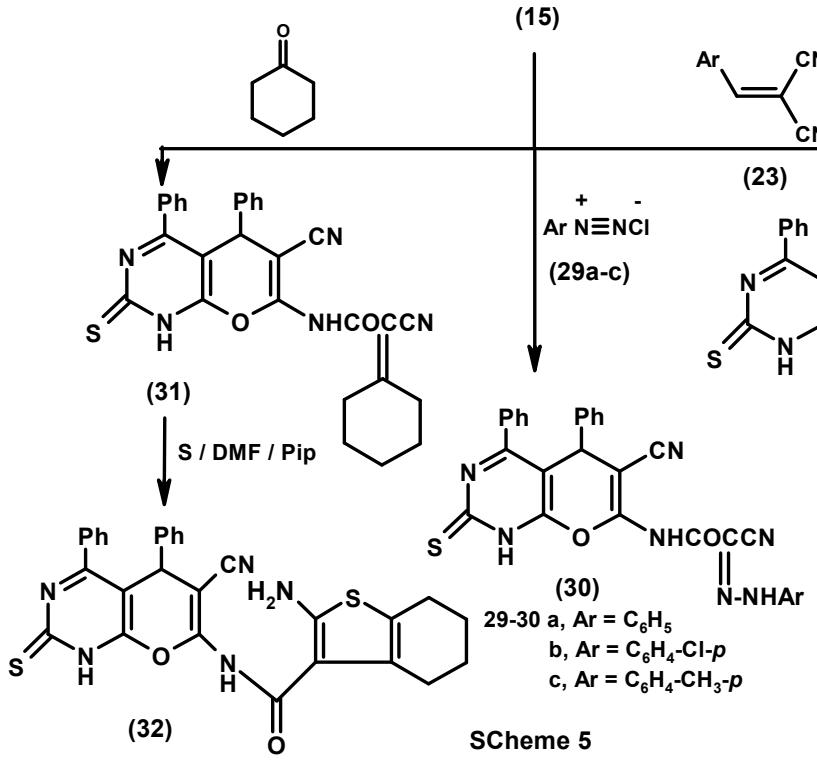


the Microanalytical Research Center, Faculty of Science, Cairo University.

Procedure for the preparation of compound (3). A mixture of 1 a (3.92 g; $0.01 \mathrm{~mol})$ and acetic anhydride $(15 \mathrm{~mL})$ was heated under reflux for $3 \mathrm{hrs}$. The reaction mixture was evaporated in vacuo and triturated with ethanol. The separated solid was filtered, washed with ether and crystallized from the proper solvent to give (3).

N-acetyl-N-(5-(4-chlorophenyl)-6cyano-4-phenyl-2-thioxo-2,5-dihydro1H-pyrano[2,3-d]pyrimidin-7-

yl)acetamide (3). Formed as brown crystals from ethanol; yield $(69 \%)$; M.p. $112-114^{\circ} \mathrm{C}$; IR $(\mathrm{KBr}) \vee \mathrm{cm}^{-1}=3215$ (NH), 3064 (CH-arom), 2934 (CH-aliph), $2199(\mathrm{CN}), 1711(\mathrm{CO}) \mathrm{cm}^{-1}$; ${ }^{1} \mathrm{H}-\mathrm{NMR}$ $\left(\mathrm{DMSO}_{-} \mathrm{d}_{6}\right) \delta=2.47\left(\mathrm{~s}, 3 \mathrm{H}, \mathrm{CH}_{3}\right), 2.48(\mathrm{~s}$, $\left.3 \mathrm{H}, \mathrm{CH}_{3}\right), 4.20$ (s, 1H, 4H-pyrane), 7.20$7.71(\mathrm{~m}, 9 \mathrm{H}$, aromatic $\mathrm{H}), 10.20(\mathrm{~s}, 1 \mathrm{H}$, $\mathrm{NH})$; MS: m/z (\%) $476\left(\mathrm{M}^{+}\right)$, Anal. Calcd. For $\mathrm{C}_{24} \mathrm{H}_{17} \mathrm{ClN}_{4} \mathrm{O}_{3} \mathrm{~S}$ (476): $\mathrm{C}, 60.44 ; \mathrm{H}$, 3.59 ; N, 11.75; Found: C, 60.45; H, 3.61; $\mathrm{N}, 11.76 \%$.

procedure for the preparation of compound (5a,b). Method (A): A mixture of 1a $(3.92 \mathrm{~g} ; 0.01 \mathrm{~mol})$ and acetic anhydride $(15 \mathrm{~mL})$ was heated under reflux for $5 \mathrm{Min}$. The reaction mixture was evaporated and allowed to cool. The separated solid was filtered, washed with water and crystallized from the proper solvent to give (5a). Method (B): A mixture of $1 \mathrm{a}(3.92 \mathrm{~g} ; 0.01 \mathrm{~mol})$ and acetyl chloride $\mathbf{4 a}$, benzoyl chloride 4b $(0.01 \mathrm{~mol})$ in pyridine $(20 \mathrm{~mL})$ was heated under reflux for $3 \mathrm{hrs}$. The reaction mixture was allowed to cool and poured into crushed ice then acidified with $\mathrm{HCl}$. The separated solid was filtered, washed with ether and crystallized from the proper solvent to give $\mathbf{( 5 a}, \mathbf{b})$.

\section{5-(4-chlorophenyl)-6-cyano-4-} phenyl-2-thioxo-2, 5-dihydro-1Hpyrano $[2,3-d]$ pyrimidin-7-yl)acetamide (5a). Formed as brown crystals from ethanol; yield (72\%); M.p.180-182 ${ }^{\circ} \mathrm{C}$; IR $(\mathrm{KBr}) \vee \mathrm{cm}^{-1}=3454,3234(2 \mathrm{NH}), 3065$ (CH-arom), 2924 (CH-aliph), $2190(\mathrm{CN})$, $1710(\mathrm{CO}) \mathrm{cm}^{-1} ;{ }^{1} \mathrm{H}-\mathrm{NMR}\left(\mathrm{DMSO}-\mathrm{d}_{6}\right) \delta=$ $1.91\left(\mathrm{~s}, 3 \mathrm{H}, \mathrm{CH}_{3}\right), 4.30(\mathrm{~s}, 1 \mathrm{H}, 4 \mathrm{H}-$ pyrane), $7.30-8.60(\mathrm{~m}, 11 \mathrm{H}$, aromatic $\mathrm{H}$ and 2NH); MS: $\mathrm{m} / \mathrm{z}(\%) 436\left(\mathrm{M}^{+}+2\right)$, Anal. Calcd. For $\mathrm{C}_{22} \mathrm{H}_{15} \mathrm{ClN}_{4} \mathrm{O}_{2} \mathrm{~S}$ (434): C, 60.76; H, 3.48; N, 12.88; Found: C, $60.78 ; \mathrm{H}, 3.89 ; \mathrm{N}, 12.90 \%$.

\section{N-(5-(4-chlorophenyl)-6-cyano-4-} phenyl-2-thioxo-2,5-dihydro-1Hpyrano[2,3-d]pyrimidin-7-yl)benzamide (5b). Formed as brown crystals from ethanol; yield (74\%); M.p.160-162 ${ }^{\circ} \mathrm{C}$; IR $(\mathrm{KBr}) \vee \mathrm{cm}^{-1}=3471(\mathrm{NH}), 3060(\mathrm{CH}-$ arom), 2925 (CH-aliph), $2216(\mathrm{CN}), 1713$ (CO) $\mathrm{cm}^{-1} ;{ }^{1} \mathrm{H}-\mathrm{NMR}\left(\mathrm{CDCl}_{3}\right) \delta=4.38(\mathrm{~s}$, $1 \mathrm{H}, \quad 4 \mathrm{H}-$ pyrane $), \quad 7.26-8.10 \quad(\mathrm{~m}, 16 \mathrm{H}$, aromatic $\mathrm{H}$ and $2 \mathrm{NH})$; MS: $\mathrm{m} / \mathrm{z}(\%) 498$ $\left(\mathrm{M}^{+}+2\right)$, Anal. Calcd. For $\mathrm{C}_{27} \mathrm{H}_{17} \mathrm{ClN}_{4} \mathrm{O}_{2} \mathrm{~S}$ (496): C, 65.25; H, 3.45; N, 11.27; Found: C, $65.24 ; \mathrm{H}, 3.43 ; \mathrm{N}, 11.25 \%$.

procedure for the preparation of compound $(6,8,10$ and 12$)$. A mixture of $1 \mathrm{a}(3.92 \mathrm{~g} ; 0.01 \mathrm{~mol})$ and hydrazine hydrate, benzene-1,2-diamine, urea and ammonium thiocyanate $(0.01 \mathrm{~mol})$ respectively in ethanol $(30 \mathrm{~mL})$ containing catalytic amount of pipridine was heated under reflux for $12 \mathrm{hrs}$. The reaction mixture was allowed to cool and poured into crushed ice then acidified with $\mathrm{HCl}$. The separated solid was filtered, washed with water and crystallized from the proper solvent to give $(6,8,10$ and 12) respectively.

\section{5-(4-chlorophenyl)-7-hydrazinyl-4- phenyl-2-thioxo-2,5-dihydro-1H-} pyrano[2,3-d]pyrimidine-6-carbonitrile (6). Formed as pale yellow crystals from ethanol; yield (83\%); M.p.100-102 ${ }^{\circ}$ C; IR $(\mathrm{KBr}) \vee \mathrm{cm}^{-1}=3323,3194\left(\mathrm{NH}_{2}\right), 2984$ (CH-aliph), 2195 (CN) cm ${ }^{-1}$; ${ }^{1} \mathrm{H}-\mathrm{NMR}$ (DMSO-d 6 ) $\delta=4.20$ (s, 1H, 4H-pyrane), $5.20\left(\mathrm{~s}, 2 \mathrm{H}, \mathrm{NH}_{2}\right), 7.03-7.83(\mathrm{~m}, 9 \mathrm{H}$, 
aromatic $\mathrm{H}), 8.67(\mathrm{~s}, 1 \mathrm{H}, \mathrm{NH}), 9.80(\mathrm{~s}$, 1H, NH); MS: m/z (\%) $408\left(\mathrm{M}^{+}+1\right)$, Anal. Calcd. For $\mathrm{C}_{20} \mathrm{H}_{14} \mathrm{ClN}_{5} \mathrm{OS}$ (407.06): $\mathrm{C}$, 58.89; H, 3.46; N, 17.17; Found: C, 58.90; H, 3.47; N, $17.19 \%$.

\section{7-(2-aminophenylamino)-5-(4-} chlorophenyl) -4-phenyl-2-thioxo-2,5dihydro-1H-pyrano[2,3-d]pyrimidine-6carbonitrile (8). Formed as pale yellow crystals from ethanol; yield $(80 \%)$; M.p. $140-142^{\circ} \mathrm{C}$; IR $(\mathrm{KBr}) \vee \mathrm{cm}^{-1}=2930$ (CH-aliph), 2197 (CN) cm ${ }^{-1}$; ${ }^{1} \mathrm{H}-\mathrm{NMR}$ $\left(\mathrm{CDCl}_{3}\right) \delta=4.12$ (s, 1H, 4H-pyrane), $5.35\left(\mathrm{~s}, 2 \mathrm{H}, \mathrm{NH}_{2}\right), 7.10-8.00(\mathrm{~m}, 14 \mathrm{H}$, aromatic $\mathrm{H}$ and $\mathrm{NH}), 8.80(\mathrm{~s}, 1 \mathrm{H}, \mathrm{NH})$; MS: m/z (\%) $485\left(\mathrm{M}^{+}+2\right)$, Anal. Calcd. For $\mathrm{C}_{26} \mathrm{H}_{18} \mathrm{ClN}_{5} \mathrm{OS}$ (483): C, 64.52; H, 3.75; N, 14.47; Found: C, 64.53; H, 3.77; $\mathrm{N}, 14.48 \%$.

\section{5-(4-chlorophenyl)-6-cyano-4- phenyl-2-thioxo-2,5-dihydro-1H- pyrano[2,3-d]pyrimidine-7-yl}

carbamimidate (10). Formed as orange crystals from ethanol; yield (80\%); M.p. $122-124^{\circ} \mathrm{C}$; IR (KBr) $\vee \mathrm{cm}^{-1}=3446$, $3351\left(\mathrm{NH}_{2}\right), 3214(\mathrm{NH}), 3065(\mathrm{CH}$-arom), 2932 (CH-aliph), 2190 (CN), 1625 (CO) $\mathrm{cm}^{-1} ;{ }^{1} \mathrm{H}-\mathrm{NMR}\left(\mathrm{CDCl}_{3}\right) \delta=4.14(\mathrm{~s}, 1 \mathrm{H}$, 4H-pyrane), 5.35 (s, 2H, $\mathrm{NH}_{2}$ ), 7.11-7.92 (m, 9H, aromatic $\mathrm{H}$ and $2 \mathrm{NH}$ ); $\mathrm{MS}: \mathrm{m} / \mathrm{z}$ (\%) $435\left(\mathrm{M}^{+}\right)$, Anal. Calcd. For $\mathrm{C}_{21} \mathrm{H}_{14} \mathrm{ClN}_{5} \mathrm{O}_{2} \mathrm{~S}$ (435): C, 57.86; H, 3.24; N, 16.07; Found: C, 57.87; H, 3.25; N, $16.08 \%$.

5-(4-chlorophenyl)-6-cyano-4-phenyl -2-thioxo-2, 5-dihydro-1H-pyrano[2,3d]pyrimidin-7-yl carbamimidothioate (12). Formed as pale yellow crystals from ethanol; yield (86\%); M.p.130-132 ${ }^{\circ} \mathrm{C}$; IR $(\mathrm{KBr}) \vee \mathrm{cm}^{-1}=3454,3369\left(\mathrm{NH}_{2}\right), 3327$, 3186 (2NH), 2938 (CH-aliph), 2214 (CN) $\mathrm{cm}^{-1} ;{ }^{1} \mathrm{H}-\mathrm{NMR}\left(\mathrm{DMSO}_{-} \mathrm{d}_{6}\right) \delta=4.80(\mathrm{~s}$, $1 \mathrm{H}, 4 \mathrm{H}$-pyrane $), \quad 7.30-7.87(\mathrm{~m}, 11 \mathrm{H}$, aromatic $\mathrm{H}$ and $\left.\mathrm{NH}_{2}\right), 10.00(\mathrm{~s}, 1 \mathrm{H}, \mathrm{NH})$, 11.10 (s, 1H, NH); MS: m/z (\%) 453 $\left(\mathrm{M}^{+}+2\right)$, Anal. Calcd. For $\mathrm{C}_{21} \mathrm{H}_{14} \mathrm{ClN}_{5} \mathrm{OS}_{2}$
(451): C, 55.81; H, 3.12; N, 15.50; Found: C, 55.82; H, 3.13; N, $15.52 \%$.

procedure for the preparation of compound (7). Method (A): A solution of 6 in sodium ethoxide $(30 \mathrm{~mL})$ was heated under reflux for $12 \mathrm{hrs}$. The solution was allowed to cool and poured into crushed ice then acidified with $\mathrm{HCl}$. The separated solid was filtered, washed with water and crystallized from the proper solvent to give (7). Method (B): A mixture of $1 \mathrm{a}(3.92 \mathrm{~g} ; 0.01 \mathrm{~mol})$, hydroxylamine hydrochloride in glacial acetic acid $(30 \mathrm{~mL})$ containing anhydrous sodium acetate $(1 \mathrm{~g})$ was heated under reflux for $24 \mathrm{hrs}$. The reaction mixture was allowed to cool and poured into cold water $(60 \mathrm{ml})$. The separated solid was filtered and crystallized to give (7).

\section{4-(4-chlorophenyl)3-methyl-5-}

phenyl-4,8-dihydropyrazolo $\left[4^{\prime}, 3^{\prime}: 5,6\right]$ pyrano[2,3-b]pyridine-7(2H)-thione (7). Formed as brown crystals from ethanol; yield (77\%); M.p.126-128 ${ }^{\circ} \mathrm{C}$; IR (KBr) v $\mathrm{cm}^{-1}=3455,3400\left(\mathrm{NH}_{2}\right), 3056(\mathrm{CH}-$ arom) $\mathrm{cm}^{-1}$; ${ }^{1} \mathrm{H}-\mathrm{NMR}$ (DMSO-d $\mathrm{d}_{6}$ ) $\delta=$ $4.20(\mathrm{~s}, 1 \mathrm{H}, 4 \mathrm{H}-$ pyrane $), 5.20(\mathrm{~s}, 2 \mathrm{H}$, $\left.\mathrm{NH}_{2}\right), 7.24-8.60(\mathrm{~m}, 11 \mathrm{H}$, aromatic $\mathrm{H}$ and $2 \mathrm{NH}$ ); Anal. Calcd. For $\mathrm{C}_{20} \mathrm{H}_{14} \mathrm{ClN}_{5} \mathrm{OS}$ (407): C, 58.89; H, 3.46; N, 17.17; Found: C, 58.90; H, 3.47; N, $17.19 \%$.

procedure for the preparation of compound $(9,11$ and 13). A solution of 8, 10 and 12 in sodium ethoxide $(30 \mathrm{~mL})$ was heated under reflux for $12 \mathrm{hrs}$. The solution was allowed to cool and poured into crushed ice then acidified with $\mathrm{HCl}$. The separated solid was filtered, washed with water and crystallized from the proper solvent to give $(9,11$ and 13).

13-(4-chlorophenyl)-1-phenyl-2thioxo-pyrimidino[b]chromeno[2,3e][1,4]diazpin-12-amine (9). Formed as brown crystals from ethanol; yield (79\%); M.p. $160-162^{\circ} \mathrm{C}$; IR $(\mathrm{KBr}) \vee \mathrm{cm}^{-1}=3384$, $3102\left(\mathrm{NH}_{2}\right), 3061$ (CH-arom), $2924(\mathrm{CH}-$ 
SINAI Journal of Applied Sciences (ISSN: 2314-6079), Vol.:(3), Is.:(2), Aug. 2014

aliph) $\mathrm{cm}^{-1} ;{ }^{1} \mathrm{H}-\mathrm{NMR}\left(\mathrm{CDCl}_{3}\right) \delta=4.40(\mathrm{~s}$, $1 \mathrm{H}, 4 \mathrm{H}$-pyrane), 5.40 (s, 2H, $\left.\mathrm{NH}_{2}\right), 7.28-$ $7.92(\mathrm{~m}, 15 \mathrm{H}$, aromatic $\mathrm{H}$ and $2 \mathrm{NH})$;

4-amino-5-(4-chlorophenyl)-6phenyl-8-thioxo-3,5,8,9-tetrahydro-2Hprimido- $\left[5^{\prime}, 4^{\prime}: 5,6\right]$ pyrano $[2,3-$

d]pyrimidin-2-one (11). Formed as brown crystals from ethanol; yield (79\%); M.p. $164-166^{\circ} \mathrm{C}$; IR (KBr) $v \mathrm{~cm}^{-1}=3452$, $3400\left(\mathrm{NH}_{2}\right), 3060$ (CH-arom), 2924 (CHaliph), 1689 (CO) $\mathrm{cm}^{-1} ; \quad{ }^{1} \mathrm{H}-\mathrm{NMR}$ $\left(\mathrm{DMSO}_{6}\right) \delta=4.30$ (s, 1H, 4H-pyrane), $6.20\left(\mathrm{~s}, 2 \mathrm{H}, \mathrm{NH}_{2}\right), 7.21-8.60(\mathrm{~m}, 11 \mathrm{H}$, aromatic $\mathrm{H}$ and $2 \mathrm{NH}$ ); Anal. Calcd. For $\mathrm{C}_{21} \mathrm{H}_{14} \mathrm{ClN}_{5} \mathrm{O}_{2} \mathrm{~S}$ (435): C, 57.86; H, 3.24; N, 16.07; Found: C, 57.87; H, 3.26; N, $16.08 \%$.

\section{6-amino-5-(4-chlorophenyl)-4-phenyl-} 5,7-dihydro-2H-pyrimido[5',4':5,6]pyrano[2,3-d]pyrimidine-2,8(1H)-dithione

Formed as pale yellow crystals from ethanol; yield (71\%); M.p.150-152 ${ }^{\circ} \mathrm{C}$; IR $(\mathrm{KBr}) \vee \mathrm{cm}^{-1}=3440,3400\left(\mathrm{NH}_{2}\right), 3061$ (CH-arom), 2920 (CH-aliph) $\mathrm{cm}^{-1} ;{ }^{1} \mathrm{H}-$ NMR $\left(\right.$ DMSO-d $\left._{6}\right) \delta=4.20(\mathrm{~s}, 1 \mathrm{H}, 4 \mathrm{H}-$ pyrane), 5.60 (s, $2 \mathrm{H}, \mathrm{NH}_{2}$ ), 7.45-8.80 (m, $11 \mathrm{H}$, aromatic $\mathrm{H}$ and $2 \mathrm{NH}$ ); Anal. Calcd. For $\mathrm{C}_{21} \mathrm{H}_{14} \mathrm{ClN}_{5} \mathrm{OS}_{2}$ (451): C, 55.81; $\mathrm{H}$, 3.12; N, 15.50; Found: C, 55.82; H, 3.13; N, $15.52 \%$.

procedure for the preparation of compound (15). A mixture of $1 \mathrm{~b}$ (3.58 g; $0.01 \mathrm{~mol})$ and ethylcyanoacetate $(0.01$ $\mathrm{mol})$ in sodium ethoxide $(30 \mathrm{~mL})$ was heated under reflux for $6 \mathrm{hrs}$. The reaction mixture was allowed to cool and poured into crushed ice then acidified with $\mathrm{HCl}$. The separated solid was filtered, washed with water and crystallized from ethanol to give (15).

2-cyano-N-(6-cyano-4,5-diphenyl-2thioxo-2,5-dihydro-1H-pyrano[2,3d]pyrimidin-7- yl)acetamide (15). Formed as yellow crystals from ethanol; yield (88\%); M.p. $180-182^{\circ} \mathrm{C}$; IR (KBr) $\vee \mathrm{cm}^{-1}=$ 3462, 3333 (2NH), 3059 (CH-arom), 2926 (CH-aliph), 2215, $2189(2 \mathrm{CN}), 1736(\mathrm{CO})$
Anal. Calcd. For $\mathrm{C}_{26} \mathrm{H}_{18} \mathrm{ClN}_{5} \mathrm{OS}$ (483): C, 64.52; H, 3.75; N, 14.47; Found: C, 64.53; $\mathrm{H}, 3.76$; N, $14.48 \%$.

$\mathrm{cm}^{-1} ;{ }^{1} \mathrm{H}-\mathrm{NMR}$ (DMSO-d $\left.{ }_{6}\right) \delta=3.72(\mathrm{~s}$, $\left.2 \mathrm{H}, \mathrm{CH}_{2}\right), 4.14$ (s, 1H, 4H-pyrane), 7.06$7.95(\mathrm{~m}, 11 \mathrm{H}$, aromatic $\mathrm{H}), 9.70(\mathrm{~s}, 1 \mathrm{H}$, $\mathrm{NH}), 10.00$ (s, 1H, NH); MS: m/z (\%) 425 $\left(\mathrm{M}^{+}\right)$, Anal. Calcd. For $\mathrm{C}_{23} \mathrm{H}_{15} \mathrm{~N}_{5} \mathrm{O}_{2} \mathrm{~S}_{2}$ (425): C, 64.93; H, 3.55; N, 16.46; Found: C, 64.94; H, 3.56; N, $16.47 \%$.

procedure for the preparation of compound (16). A mixture of 15 (4.25 g; $0.01 \mathrm{~mol})$ and DMF-DMA $(0.01 \mathrm{~mol})$ in dioxane $(50 \mathrm{~mL})$ was heated under reflux for $6 \mathrm{hrs}$. The reaction mixture was evaporated in vacuo and triturated with ethanol. The separated solid was filtered, washed with ether and crystallized from the proper solvent to give (16).

2-cyano-N-(6-cyano-4,5-diphenyl-2thioxo-2,5-dihydro-1H-pyrano[2,3d]pyrimidin-7-yl)-3-

(dimethylamino)acrylamide (16). Formed as yellow crystals from ethanol; yield $(80 \%)$; M.p. $100-102^{\circ} \mathrm{C}$; IR (KBr) $v \mathrm{~cm}^{-1}=3332$, 3211 (2NH), 3059 (CH-arom), 2923 (CHaliph), 2212, $2192 \quad$ (2CN), 1733 (CO) $\mathrm{cm}^{-1} ;{ }^{1} \mathrm{H}-\mathrm{NMR}\left(\mathrm{CDCl}_{3}\right) \delta=3.22(\mathrm{~s}, 3 \mathrm{H}$, $\left.\mathrm{CH}_{3}\right), 3.25\left(\mathrm{~s}, 3 \mathrm{H}, \mathrm{CH}_{3}\right), 3.80(\mathrm{~s}, 1 \mathrm{H}, 4 \mathrm{H}-$ pyrane), 7.27-7.58 (m, $11 \mathrm{H}$, aromatic $\mathrm{H}$ and $\mathrm{CH}$-oliffin), 7.99 (s, 1H, NH), 8.80 (s, 1H, NH); MS: m/z (\%) $480\left(\mathrm{M}^{+}\right)$, Anal. Calcd. For $\mathrm{C}_{26} \mathrm{H}_{20} \mathrm{~N}_{6} \mathrm{O}_{2} \mathrm{~S}$ (480): C, 64.98; H, 4.20; N, 17.49; Found: C, 64.99; H, $4.21 ; \mathrm{N}, 17.51 \%$.

procedure for the preparation of compound (17). A mixture of 16 (4.80 g; $0.01 \mathrm{~mol})$ and hydrazine hydrate $(0.01$ $\mathrm{mol})$ in ethanol $(50 \mathrm{~mL})$ was heated under reflux for $8 \mathrm{hrs}$. The reaction mixture was left to stand and poured into water then acidified with $\mathrm{HCl}$. The separated solid was filtered, washed with water and crystallized from the proper solvent to give (17).

5-amino-N-(6-cyano-4,5-diphenyl-2thioxo-2, 5-dihydro-1H-pyrano [2,3-d] 
pyrimidin-7-yl)-1H-pyrazole-4-

carboxamide (17). Formed as yellow crystals from ethanol; yield (71\%); M.p. $150-152^{\circ} \mathrm{C}$; IR $(\mathrm{KBr}) \vee \mathrm{cm}^{-1}=3331$, $3207\left(\mathrm{NH}_{2}\right), 3060$ (CH-arom), $2923(\mathrm{CH}-$ aliph), 2208 (CN) cm ${ }^{-1}$; ${ }^{1} \mathrm{H}-\mathrm{NMR}$ (DMSO-d 6$) \delta=4.20$ (s, 1H, 4H-pyrane), $5.80\left(\mathrm{~s}, 2 \mathrm{H}, \mathrm{NH}_{2}\right), 7.21-8.30(\mathrm{~m}, 14 \mathrm{H}$, aromatic $\mathrm{H}, 3 \mathrm{NH}$ and $\mathrm{CH}$-oliffin); MS: $\mathrm{m} / \mathrm{z} \quad(\%) 467 \quad\left(\mathrm{M}^{+}\right)$, Anal. Calcd. For $\mathrm{C}_{24} \mathrm{H}_{17} \mathrm{~N}_{7} \mathrm{O}_{2} \mathrm{~S}$ (467): C, 61.66; H, 3.67; N, 20.97; Found: C, 61.67; H, 3.69; N, 20.98 $\%$.

procedure for the preparation of compound (22). A mixture of 16 (4.80 g; $0.01 \mathrm{~mol})$ and malononitrile $(0.01 \mathrm{~mol})$ in ethanol $(30 \mathrm{~mL})$ containing catalytic amount of piperidine was heated under reflux for $24 \mathrm{hrs}$. The reaction mixture was allowed to cool and poured into crushed ice then acidified with $\mathrm{HCl}$. The separated solid was filtered, washed with water and crystallized from the proper solvent to give (22).

\section{5-Amino-N-(6-cyano-4,5-diphenyl-2- thioxo-2,5-dihydro-1H-pyrano[2,3- d]pyrimidin-7-yl)-1H-pyrazole-4-}

carboxamide (22). Formed as yellow crystals from ethanol; yield (74\%); M.p. $162-164^{\circ} \mathrm{C}$; IR $(\mathrm{KBr}) \vee \mathrm{cm}^{-1}=3150$ (NH), $2196(\mathrm{CN}), 1639(\mathrm{CO}) \mathrm{cm}^{-1} ;{ }^{1} \mathrm{H}-$ NMR $\left(\right.$ DMSO-d $\left._{6}\right) \delta=3.81(\mathrm{~s}, 1 \mathrm{H}, 4 \mathrm{H}-$ pyrane), 7.23-7.95 (m, $14 \mathrm{H}$, aromatic $\mathrm{H}$, $3 \mathrm{NH}$ and $\mathrm{CH}-$ oliffin); MS: $\mathrm{m} / \mathrm{z}$ (\%) 503 $\left(\mathrm{M}^{+}+2\right)$, Anal. Calcd. For $\mathrm{C}_{27} \mathrm{H}_{15} \mathrm{~N}_{7} \mathrm{O}_{2} \mathrm{~S}$ (501): C, 64.66; H, 3.01; N, 19.55; Found: C, 64.67; H, 3.03; N, $19.56 \%$.

procedure for the preparation of
compound (28). A mixture of dihydropyrimidinone derivatives $\mathbf{1 5}$ (4.25 $\mathrm{g} ; 0.01 \mathrm{~mol})$ and 4-chlorobenzylidenemalononitrile $23(0.01 \mathrm{~mol})$ in ethanol (30 $\mathrm{mL}$ ) containing catalytic amount of piperidine was heated under reflux for 24 hrs. The reaction mixture was allowed to cool and poured into crushed ice then acidified with $\mathrm{HCl}$. The separated solid was filtered, washed with water and crystallized from the proper solvent to give (28).

7-Amino-4, 5-diphenyl-2-thioxo-2, 5dihydro-1H-pyrano[2,3-d]pyrimidine-6carbonitrile (28). Formed as white crystals from ethanol; yield (87\%); M.p. $130-132^{\circ} \mathrm{C}$; IR (KBr) $\vee \mathrm{cm}^{-1}=3453$, 3331, 3210 (3NH), 3062 (CH-arom), 2929 (CH-aliph), $2192(\mathrm{CN}), 1626(\mathrm{CO}) \mathrm{cm}^{-1}$; ${ }^{1} \mathrm{H}-\mathrm{NMR}\left(\mathrm{CDCl}_{3}\right) \delta=3.13-3.15(\mathrm{~d}, 1 \mathrm{H}$, CH) $\mathrm{J}=6.00 \mathrm{~Hz}, 3.77-3.81(\mathrm{~d}, 1 \mathrm{H}, \mathrm{CH}) \mathrm{J}$ $=12.00 \mathrm{~Hz}, 4.48(\mathrm{~s}, 1 \mathrm{H}, 4 \mathrm{H}-$ pyrane$)$, 7.02-7.94 (m, $17 \mathrm{H}$, aromatic $\mathrm{H}$ and $3 \mathrm{NH})$; MS: m/z (\%) $614\left(\mathrm{M}^{+}+1\right)$, Anal. Calcd. For $\mathrm{C}_{33} \mathrm{H}_{20} \mathrm{ClN}_{7} \mathrm{O}_{2} \mathrm{~S}$ (613): C, 64.54; H, 3.28; N, 15.97; Found: C, 64.55; H, 3.29; N, $15.99 \%$.

procedure for the preparation of compound (30a-c). A cold suspension of aryl diazonium salts $(0.002 \mathrm{~mol})$ (prepared from $0.002 \mathrm{~mol}$ of aromatic amine with the appropriate quantities of sodium nitrite and hydrochloric acid) was gradually added to a cold solution $\left(0-5^{\circ} \mathrm{C}\right)$ of $15(0.002 \mathrm{~mol})$ in ethanol $(50 \mathrm{~mL})$ containing anhydrous sodium acetate $(5 \mathrm{~g})$ with continuous stirring for $1 \mathrm{hr}$. The resulting reaction product was filtered off, washed with water and crystallized from the proper solvent to give compounds (30a-c).

\section{2-(6-cyano-4,5-diphenyl-2-thioxo-2,5-} dihydro-1H-pyrano[2,3-d]pyrimidin-7ylamino)-2-oxo-N'-phenylacetohydrazonoyl cyanide (30a). Formed as orange crystals from ethanol; yield (86\%); M.p.110$112^{\circ} \mathrm{C}$; IR $(\mathrm{KBr}) \vee \mathrm{cm}^{-1}=3439,3384$ (2NH), 3061 (CH-arom), 2930 (CHaliph), $2210(\mathrm{CN}), 1631(\mathrm{CO}) \mathrm{cm}^{-1}$; ${ }^{1} \mathrm{H}-$ NMR $\left(\mathrm{CDCl}_{3}\right) \delta=4.40 \quad(\mathrm{~s}, 1 \mathrm{H}, 4 \mathrm{H}-$ pyrane), 7.20-7.80 (m, $18 \mathrm{H}$, aromatic $\mathrm{H}$ and $3 \mathrm{NH}) ; \mathrm{MS}: \mathrm{m} / \mathrm{z}(\%) 529\left(\mathrm{M}^{+}\right)$, Anal. Calcd. For $\mathrm{C}_{29} \mathrm{H}_{19} \mathrm{~N}_{7} \mathrm{O}_{2} \mathrm{~S}$ (529): C, 65.77; H, 3.62; N, 18.51; Found: C, 65.78; H, $3.63 ; \mathrm{N}, 18.52$.

N'-(4-chlorophenyl)-2-(6-cyano-4,5 diphenyl-2-thioxo-2, 5-dihydro-1H-pyrano [2,3-d]pyrimidin-7-ylamino)-2-oxoaceto- 
hydrazonoyl cyanide (30b). Formed as orange crystals from ethanol; yield $(83 \%)$; M.p. $114-116^{\circ} \mathrm{C}$; IR $(\mathrm{KBr}) \vee \mathrm{cm}^{-1}=3442$, 3181 (2NH), 3063 (CH-arom), 2925 (CHaliph), $2212(\mathrm{CN}), 1639(\mathrm{CO}) \mathrm{cm}^{-1} ;{ }^{1} \mathrm{H}-$ NMR $\left(\right.$ DMSO-d $\left._{6}\right) \delta=4.20(\mathrm{~s}, 1 \mathrm{H}, 4 \mathrm{H}-$ pyrane), 7.23-8.20 (m, $17 \mathrm{H}$, aromatic $\mathrm{H}$ and $3 \mathrm{NH}) ; \mathrm{MS}: \mathrm{m} / \mathrm{z}(\%) 563\left(\mathrm{M}^{+}\right)$, Anal. Calcd. For $\mathrm{C}_{29} \mathrm{H}_{18} \mathrm{ClN}_{7} \mathrm{O}_{2} \mathrm{~S}$ (563): $\mathrm{C}$, 61.76; H, 3.22; N, 17.38; Found: C, 61.77; H, 3.23; N, $17.40 \%$.

2-(6-cyano-4,5-diphenyl-2-thioxo-2,5dihydro-1H-pyrano[2,3-d]pyrimidin-7ylamino)-2-oxo-N'-p-

tolylacetohydrazonoyl cyanide $(30 \mathrm{c})$. Formed as orange crystals from ethanol; yield (80\%); M.p.122-124 ${ }^{\circ} \mathrm{C}$; IR ( $\left.\mathrm{KBr}\right)$ v $\mathrm{cm}^{-1}=3452,3378(2 \mathrm{NH}), 3062(\mathrm{CH}-$ arom), 2931 (CH-aliph), $2212(\mathrm{CN}), 1629$ (CO) $\mathrm{cm}^{-1} ;{ }^{1} \mathrm{H}-\mathrm{NMR}\left(\mathrm{CDCl}_{3}\right) \delta=2.09(\mathrm{~s}$, $\left.3 \mathrm{H}, \mathrm{CH}_{3}\right), 4.30(\mathrm{~s}, 1 \mathrm{H}, 4 \mathrm{H}-$ pyrane $), 7.08-$ $8.00(\mathrm{~m}, 17 \mathrm{H}$, aromatic $\mathrm{H}$ and $3 \mathrm{NH})$; MS: $\mathrm{m} / \mathrm{z} \quad(\%) 543\left(\mathrm{M}^{+}\right)$, Anal. Calcd. For $\mathrm{C}_{30} \mathrm{H}_{21} \mathrm{~N}_{7} \mathrm{O}_{2} \mathrm{~S}$ (543): C, 66.28; H, 3.89; N, 18.04; Found: C, 66.29; H, 3.90; N, 18.05 $\%$.

procedure for the preparation of compound (31). A mixture of dihydropyrimidinone derivatives $\mathbf{1 5}$ (4.25 g; $0.01 \mathrm{~mol})$ and cyclohexanone $(0.01$ mol) in ethanol $(50 \mathrm{~mL})$ containing catalytic amount of piperidine was heated under reflux for $6 \mathrm{hrs}$. The reaction mixture was allowed to cool and poured into crushed ice then acidified with $\mathrm{HCl}$. The separated solid was filtered, washed with water and crystallized from the proper solvent to give (31).

2-cyano-N-(6-cyano-4,5-diphenyl-2-

\section{thioxo-2,5-dihydro-1H-pyrano} [2,3d] pyrimidin-7-yl)-2-cyclohexlideneacetamide (31). Formed as yellow crystals from ethanol; yield (82\%); M.p.122-124 ${ }^{\circ} \mathrm{C}$; IR $(\mathrm{KBr}) \vee \mathrm{cm}^{-1}=3328,3203(2 \mathrm{NH}), 3060$ (CH-arom), 2923 (CH-aliph), $2204(\mathrm{CN})$, $1626(\mathrm{CO}) \mathrm{cm}^{-1} ;{ }^{1} \mathrm{H}-\mathrm{NMR}\left(\mathrm{CDCl}_{3}\right) \delta=$ 0.87-1.71 (m, 6H, 3CH $), 3.14-3.97(\mathrm{~m}$, $4 \mathrm{H}, 2 \mathrm{CH}_{2}$ ), 4.50 (s, 1H, 4H-pyrane), 7.27- $7.98(\mathrm{~m}, 12 \mathrm{H}$, aromatic $\mathrm{H}$ and $2 \mathrm{NH})$; MS: $\mathrm{m} / \mathrm{z}(\%) 505\left(\mathrm{M}^{+}\right)$, Anal. Calcd. For $\mathrm{C}_{29} \mathrm{H}_{23} \mathrm{~N}_{5} \mathrm{O}_{2} \mathrm{~S}$ (505): C, 68.89; H, 4.59; N, 13.85; Found: C, 68.90; H, 4.61; N, 13.86 $\%$.

procedure for the preparation of compound (32). A mixture of dihydropyrimidinone derivatives $\mathbf{3 1}$ (5.05 g; $0.01 \mathrm{~mol})$ and elemental sulfur (0.01 mol) in DMF $(50 \mathrm{~mL})$ containing catalytic amount of piperidine was heated under reflux for $12 \mathrm{hrs}$. The reaction mixture was allowed to cool and poured into crushed ice then acidified with $\mathrm{HCl}$. The separated solid was filtered, washed with water to give (32).

2-amino-N-(6-cyano-4,5-diphenyl-2thioxo-2,5-dihydro-1H-pyrano[2,3-d] pyrimidin-7-yl)-4,5,6,7-tetrahydrobenzeno [b]thiophene-3-carboxamide (32). Formed as yellow crystals from ethanol; yield (80\%); M.p. $160-162^{\circ} \mathrm{C}$; IR ( $\left.\mathrm{KBr}\right) \vee \mathrm{cm}^{-1}=$ 3435 (NH), 3084 (CH-arom), 2933 (CHaliph), $2210(\mathrm{CN}), 1713(\mathrm{CO}) \mathrm{cm}^{-1} ;{ }^{1} \mathrm{H}-$ NMR (DMSO-d $\left.{ }_{6}\right) \delta=1.23-1.60(\mathrm{~m}, 4 \mathrm{H}$, $\left.2 \mathrm{CH}_{2}\right), 3.30-3.40\left(\mathrm{~m}, 4 \mathrm{H}, 2 \mathrm{CH}_{2}\right), 3.80(\mathrm{~s}$, $1 \mathrm{H}, \quad 4 \mathrm{H}-$ pyrane $), \quad 7.23-7.80(\mathrm{~m}, 14 \mathrm{H}$, aromatic $\mathrm{H}, 2 \mathrm{NH}$ and $\left.\mathrm{NH}_{2}\right)$; $\mathrm{MS}: \mathrm{m} / \mathrm{z}(\%)$ $537\left(\mathrm{M}^{+}\right)$, Anal. Calcd. For $\mathrm{C}_{29} \mathrm{H}_{23} \mathrm{~N}_{5} \mathrm{O}_{2} \mathrm{~S}_{2}$ (537): C, 64.78; H, 4.31; N, 13.03; Found: C, 64.79; H, 4.30; N, $13.04 \%$.

\section{ACKNOWLEDGMENT}

The authors are very grateful to Prof. Dr. A. E. Khodair, Department of Chemistry, Faculty of Science, Suez Canal University, Ismailia, Egypt, for valuable support and reviewing this manuscript.

\section{REFERENCES}

Amed. A. F, Adel. A. H. A, Ezzat. A. H. and Ekbal. H. K. (2012). American Journal of organic Chemistry, 2(2): 713.

Bedair, A. H.; Nagwa. A. E.; Abd ElLatif, M. S.; A. H. Fakery and A. 
M. El-Agrody. (2000). Farmaco, 55, 708-417.

Bianchi, G. and Tava, A. (1987). Agric, Biol. Chem, 51, 2001.

Dell, C. P. and Smith, C. W. (1993). Eur. Pat. 537, 94, 9, 21 Apr 1993; Chem. Abstr. 119, 139102d.

Eiden, F. and F. Denk. (1991). Arch. Pharm. Weinheim Ger. 324, 353-354.

El-Agrody, A. M.; Abd El-Latif, M. S.; El-Hady, N. A.; Fakery, A. H. and A. H. Bedair. (2001). Molecules, 6, 519-527.

El-Agrody, A. M.; M. H. El-Hakim, M. S. El-Latif, A. H. Fakery, E. M. ElSayed and K. A. El-Ghareb. (2000). Acta Pharm, 50, 111-120.

Elden, F. and F. Denk. (1991). Arch Pharm. Weinheim Ger. 324, 353.

El-Gaby, M. S. A., S. M. Abdel-Gawad, M. M. Ghorab, H. I. Heiba and H. M. Aly. (2006). Phosphorus, Sulfur, and Silicon, 181:279-297.

El-Sharkawy, K. A., N. N. E. El-Sayed and M. Y. Zaki. (2012). International Research Journal of Pure \& Applied Chemistry, 2(1): 91-104.

Fathy. A. Eid, Ashraf. H. F. A, Gamal. A. M. E and Moustafa. M. K. (2004). Acta Pharm, 54, 13-26.

Gamal. A. E, Hanaa. H. A and Jihan. S. H. (2005). European Journal of Medical Chemistry, 40, 1283-1294.

Haider. B, Hamada. M. I, Saad. M. and Mohamed. H. E. (2012). European Journal of Medical Chemistry, 52, 5165.

Hatem. M. G, Sherif. M. S and Fathi. A. A. (2005). Journal of Sulfur Chemistry, 26, 393-403.

Hirmoto. K.; A. Nasuhara.; K. Michiloshi.; T. Kato and K. Kikugawa. (1997). Mutat. Res. 395, 47-56.
Khaled. D. K, Hamad. M. A, Doa'a. M. $A$ and Mohamed. H. E. (2009). Tetrahedron, 65, 9421-9427.

Martnez. A. G. and L. J. Marco, Bioorg, Med. (1997). Chem. Lett., 7, 3165-3170.

Mohamed. A. A. R and Mohamed. E. (2007). Bioorganic \& Medicinal Chemistry, 15, 1206-1211.

Mohammed, F. K.; A. Y. Soliman, A. Ssawy and M. G. Badre. (2009). Journal of Chemical and Pharmaceutical Research, 1(1), 213224.

Mohammed. A. B, Sayed. A. A and Mohga. M. E. (1989). Journal of Islamic Academy of Sciences 2:4, 241-243.

Ohira,T. and M. yatagai. (1993). J. Jpn, Wood Res. Soc. 39.

Saied. A. El-Asslery, Galal. H. sayed and Ahmed. F. (2004). Acta Pharm. $54,143$.

Salman, A. S. S. (1999). Commun. Fac. Sci. Univ. Ank. Series B, 45, 85-91.

Samir. B, Walid. F and Mohamed. A. M. (2009). European Journal of Medical Chemistry, 44, 4813-4818.

Sherif, S. M., A. M. Hussein. (1997). Monatshefte fur Chemie, 128, 687696.

Shishoo, C. J.; M. B. devani, G. V. Ullas, S. Ananthan, V. S. Bahadit. (1981). J. Heterocyclic Chem, 18, 4346.

Siham. A. A. (2012). Molecules, 17, 10902-10915.

Tandon, V. K.; M. Vaish, S. Jain, D. S. Bhakuni and R. C. Srimal. (1991). Indian. J. Pharm. Sci, 53, 22-23.

Taylor, R. N.; A. Cleasby, O. Singh and T. Skarzynski. (1998). J. Med. Chem, 41, 798-807. 


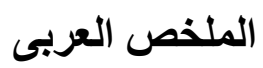

التحضيرات غير المتجانسة الحلقة مع النيتريلات النشطة: والتحضيرات المتوقعة لمشتقات عديدة

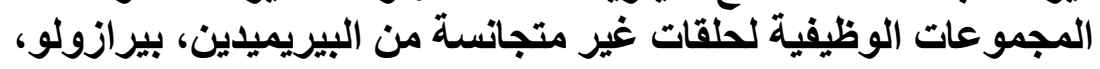

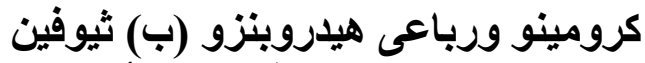

محمد أحمد عليان، إبراهيم سعد عبد الحافظ، محمد أحمد محمود عبد فئر الرحيم

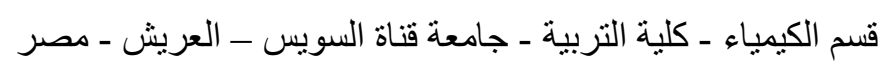

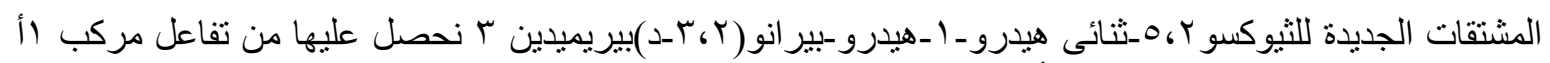

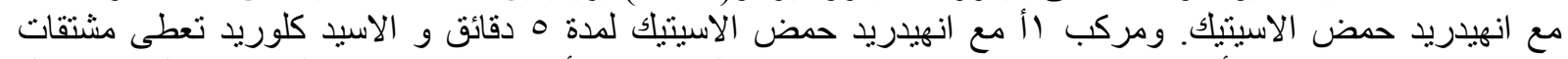

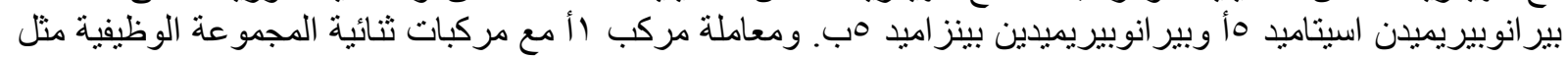

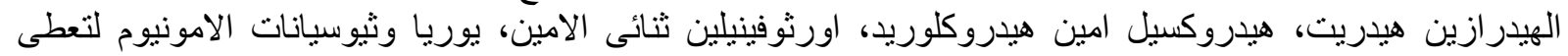

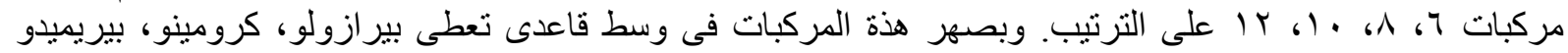

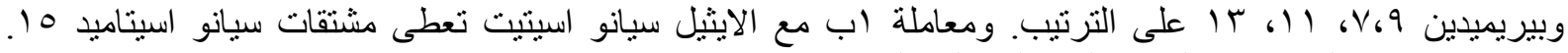

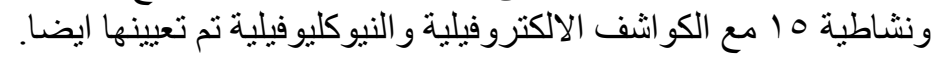

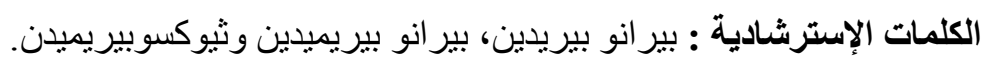


